\title{
School Choice in a Market Environment: Individual versus Social Expectations
}

\author{
Catalina Canals $\left(\mathbb{D},{ }^{1}\right.$ Eric Goles $\mathbb{D}^{1},{ }^{1}$ Aldo Mascareño $(\mathbb{D})^{2}$ \\ Sergio Rica $\left(\mathbb{D},{ }^{1}\right.$ and Gonzalo A. Ruz $\mathbb{C}^{1,3}$ \\ ${ }^{1}$ Facultad de Ingeniería y Ciencias, Universidad Adolfo Ibáñez, Av. Diagonal Las Torres 2640, Peñalolén, Santiago, Chile \\ ${ }^{2}$ Escuela de Gobierno, Universidad Adolfo Ibáñez, Av. Diagonal Las Torres 2640, Peñalolén, Santiago, Chile \\ ${ }^{3}$ Center of Applied Ecology and Sustainability (CAPES-UC), Santiago, Chile
}

Correspondence should be addressed to Gonzalo A. Ruz; gonzalo.ruz@uai.cl

Received 28 June 2018; Revised 25 October 2018; Accepted 6 November 2018; Published 2 December 2018

Academic Editor: Carlos Gershenson

Copyright (C) 2018 Catalina Canals et al. This is an open access article distributed under the Creative Commons Attribution License, which permits unrestricted use, distribution, and reproduction in any medium, provided the original work is properly cited.

\begin{abstract}
School choice is a key factor connecting personal preferences (beliefs, desires, and needs) and school offer in education markets. While it is assumed that preferences are highly individualistic forms of expectations by means of which parents select schools satisfying their internal moral standards, this paper argues that a better matching between parental preferences and school offer is achieved when individuals take into account their relevant network vicinity, thereby constructing social expectations regarding school choice. We develop two related models (individual expectations and social expectations) and prove that they are driven by a Lyapunov function, obtaining that both models converge to fixed points. Also, we assess their performance by conducting computational simulations. While the individual expectations model shows a probabilistic transition and a critical threshold below which preferences concentrate in a few schools and a significant amount of students is left unattended by the school offer, the social expectations model presents a smooth dynamics in which most of the schools have students all the time and no students are left out. We discuss our results considering key topics of the empirical research on school choice in educational market environments and conclude that social expectations contribute to improve information and lead to a better matching between school offer and parental preferences.
\end{abstract}

\section{Introduction}

As a consequence of fiscal deficits and changes in the political arena, several countries in Europe, the Americas, and other world regions began to introduce a market competitive approach in the design and organization of public services during the 1980s and 1990s [1-3]. In this shifting policy landscape, education underwent major changes worldwide, mostly related to schools' competition for students and a wider range of choice for parental selection of schools [4]. Since then, a political and scholarly debate on the publicprivate structure of school systems and its consequences has developed.

In the 1990s, for example, Chubb and Moe [5], in a highly controversial article, argued that bureaucratic control over schools must be eliminated and replaced by school market and parental choice as key factors for improving efficiency in primary and secondary education. Similarly, [6] concluded that the opportunities for individual choice in countries such as The Netherlands, England, and Scotland increased significantly because of market reforms. References $[7,8]$, on the contrary, argue that education policy evolved to combine competition and cooperation, namely, local management, pupil-led funding, parental choice, on the one side, and a core curriculum and state regulation, on the other. Likewise, [9] observes that parental choice does not directly means that families become customers or consumers in an educational market environment. Also entangled with the concept and practice of school choice are the exercise of liberal rights (the right of parents to decide the school of their children) and the operation of mechanisms of distributive justice (granting the possibility of poor families to select the same or similar options that richer families). In the last decade, the controversy remains. While some analyses emphasize that 
school choice, as a right to decide or promote distributive justice, is highly constrained by the prevailing inequities in the social environment (e.g., [10-13]), others highlight the achievements of parental choice, vouchers, and charter schools in increasing the options to choose from for families in regions with failing or low performance schools [14], in improving the general quality of the system through competition (e.g., $[15,16])$, and in achieving a better match between quality school offer and parental preferences (e.g., $[17,18])$.

More than four decades of debate regarding school choice seem to involve not only controversial scientific analyses and empirical evidence, but also contrasting epistemic standpoints and opposite political stances that reveal the complexity of modern school systems and their relevance in contemporary society $[19,20]$. In cases like this, a modeling and simulation approach to complex systems could be useful to capture the fundamental dynamics behind the historical and empirical variation that characterizes the concrete performance of school systems in different social contexts. In doing so, the analysis certainly detaches itself from particular cases or specific stories, yet it gains in abstraction and generalization to observe relevant patterns that emerge from simple rules or distinctions lying at the basis of complex phenomena.

One fundamental distinction in the school choice debate has always been the difference between institutional framework (school offer, either public, private, or hybrid, with different attributes) and selecting agents (parents or families with a clear motivation for improving their own children's outcomes). In this paper, we focus on the selecting agents and consider that the institutional framework offers, at a given time, a certain set of attributes that may or may not match with the parental set of preferences. By focusing on the selecting agents, we aim to contribute to a clearer, though mathematically stylized view on parental preferences. The debate on school choice generally assumes parental preferences as highly individualistic forms of expectations with already internalized societal values that can be directly transformed into actions and decisions [21], in this case, a school choice. While in certain cases this may work for individuals with strong preferences (beliefs, desires, and needs), social relations or particular social situations contextually change individual preferences through social influence [22] and social interactions that take place in the very process of decision-making [23].

By applying computational simulations, in this paper we concentrate on the dynamics of preferences (either individual or social) and the way they impinge upon the general complex dynamics of the school system. We propose two mathematical models of choice where the nodes of a network represent individuals and the edges interconnecting them symbolize social relations. In the first model, given an initial configuration of choices in each node of the network, individuals make a decision according to a deterministic rule of choice over a set of attributes based on two steps: first, her own preferences regarding school attributes and, in case of a tie between two or more possibilities, the most represented attribute (the majority rule) is selected. Conversely, in the second model, we analyze the opposite dynamics. We consider first the most represented attribute in the network vicinity (according to the majority rule), and only in case of a tie (between two or more attributes), we take into account the individual preference. We show that both rules of decision express two different dynamics. To that extent, it matters which decision rule is taken first, if that based on the individual or that based on the majority rule.

The majority rule model $[24,25]$ has been applied in the analysis of complex systems in fields such as biology (neural networks) [26], physics (spin glass model) [27], and social sciences (voting problems) [28-31]. The novelty in our case consists in studying more than one level decision rule (two in our article) in a social domain. Considering these two above-described models, we call the first one, the model of individual expectations, and the second one, the model of social expectations. While the former understands persons as predominantly individualistic agents who are able to compute alternatives to decide based on their own strong beliefs, desires, and needs, the latter introduces the influence of the social landscape (network vicinity) as a key factor explaining school choice decisions. The rules of decision at the basis of these models, although simple, produce nonlinear behaviors, and since individuals act in a finite network of many agents, their dynamics is in some respects unexpected and cannot be deduced from the analysis of the local behavior. The emergent patterns obtained (attractors of the dynamics) are more than the aggregation of the individual behavior of each agent. In other words, our models of school choice behave as complex systems.

Concretely, our simulations show that the stronger the individualistic view on school choice is performed by parents, the more likely is that the system presents an unstable dynamics characterized by a probabilistic transition and a critical threshold [32]. Since individual expectations are deemed stable (strong beliefs, desires, and needs), most of the individuals (parents) will select those schools whose attributes perfectly match their heterogeneous preferences, producing over population of some schools and under population of others. On the contrary, considering social expectations, no transition appears, thereby suggesting that social expectations bring about a more smooth system dynamics than individual expectations regarding the matching between school offer and parental preferences in school choice. In this case, from a priority of individual expectations (strong beliefs, desires, and needs) the dynamics of school choice moves to a priority of social expectations in which the former are adapted, even modified, according to social interactions with the network vicinity in decision-making processes [33].

In order to deploy this argument, the article proceeds as follows. First, we construct the two variants of our model of school choice (individual expectations and social expectations). Second, we consider a Potts-like energy to measure the convergence of the two strategies. Third, we run simulations for these strategies. Fourth, we connect the results of our simulations with some relevant topics in the empirical literature of school choice. And fifth, we draw some conclusions from the analysis. 


\section{The Model}

We consider two related models of school choice. We call the first one the model of individual expectations, which gives priority to personal preferences (beliefs, desires, and needs), i.e., individual choice privileges the maximum matching between preferences and school offer. The second one is called the model of social expectations. Here, priority is given to preferences of the network vicinity. We develop both models below.

2.1. Individual Expectations Dynamics. Let us consider $Q=$ $\{1,2, \ldots q\}$ the set of states. Each state $k$ is associated with a vector of attributes $\boldsymbol{a}(k)=\left(a_{1}(k), \ldots, a_{s}(k)\right) \in\{0,1\}^{s}$. Each element $a_{l}(k) \in\{0,1\}$ of this vector represents an attribute of the state $k$. That is, $a_{l}(k)=1$ if and only if, the state or school $Q_{k}$ has the attribute $l$.

Further, we consider a network defined by a graph $G=$ $(V, E)$, where $V$ represents the set of individuals and $E$ the links between them. For a network of $N$ individuals, let $\boldsymbol{x}=$ $\left(x_{1}, \ldots, x_{N}\right)$ be a state of the network where $x_{i} \in Q$. Also, let $\boldsymbol{b}(i) \in\{0,1\}^{s}$ be the expectations of the $i$-th individual. We emphasize that $k$ is a state in $Q$, and the $x_{i}$ are states in $G$.

We propose the following dynamics. Consider the asynchronous updating scheme, i.e., at each step an individual is randomly chosen and updated under the following local decision rule: given the chosen individual $i$, the state $x_{i}$, and the attribute vector: $\boldsymbol{a}\left(x_{i}\right) \in\{0,1\}^{s} \forall i$, we check from all the neighboring nodes, i.e., $\forall j \in V_{i}$, which neighbors satisfy

$$
\boldsymbol{b}(i) \cdot \boldsymbol{a}\left(x_{j}\right)>\boldsymbol{b}(i) \cdot \boldsymbol{a}\left(x_{i}\right),
$$

where the symbol - represents the inner product, i.e., $\boldsymbol{b}(i)$. $\boldsymbol{a}\left(x_{j}\right)=\sum_{l=1}^{s} b_{l}(i) \cdot a_{l}\left(x_{j}\right)$.

From all the neighbors that satisfy (1), we select randomly one of them. We call this neighbor $j^{*}$. Then, the new state for $i$ is

$$
x_{i}^{\prime}=x_{j^{*}}
$$

If no neighbor satisfies (1), then we consider neighbors satisfying the condition

$$
\boldsymbol{b}(i) \cdot \boldsymbol{a}\left(x_{j}\right)=\boldsymbol{b}(i) \cdot \boldsymbol{a}\left(x_{i}\right) .
$$

In this case, we select the winning state by majority voting, the most represented state in the neighborhood. If the winning state is associated with neighbor $j^{* *}$, then the new state for $i$ will be

$$
x_{i}^{\prime}=x_{j^{* *}}
$$

If no neighbor satisfies any of the two previous conditions, then $x_{i}$ remains the same. In case of a tie (two or more states equally represented in the neighborhood), we must consider the following: (i) if one of the states corresponds to the same state of $i$, then the winner is the state of $i$, and therefore $x_{i}$ remains the same; (ii) if the state of $i$ does not belong within the states that are tied, then the winning state is selected randomly from the tied states.
The central idea behind this updating procedure is that the new state $x_{i}^{\prime}$ fits better with the expected attributes of $i$ (condition (1)) or it is represented by the neighborhood (condition (3)).

2.2. Social Expectations Dynamics. This dynamics is similar to the previous one, yet in this case, we focus on generalized social preferences. We first apply majority voting, i.e., the most represented state in the neighborhood of the selected individual and, in case of a tie, we select a neighbor that maximizes the correlation with individual expectations.

As in the previous case, we consider the asynchronous updating scheme. The chosen individual is the most represented state in the neighborhood. For doing this, we have to consider the following quantities: $C_{i}(k)=\left|\left\{j \in V_{i} / x_{i}=x\right\}\right|$ for any $k \in Q$ and $c=\max \left(C_{i}(k) / k \in Q\right)$. If there exists at least one neighbor $j *$, such that $c=C_{i}\left(x_{j *}\right)$, then the next state of individual $i$ will be $x_{i}^{\prime}=x_{j *}$.

In case of a tie, i.e., there exists several neighbors $k$ such that $c=C_{i}\left(x_{k}\right)$, we choose one neighbor considering that

$$
\boldsymbol{b}(i) \cdot \boldsymbol{a}\left(x_{k}\right)>\boldsymbol{b}(i) \cdot \boldsymbol{a}\left(x_{i}\right) .
$$

If the previous condition does not hold, the state of individual $i$ remains unchanged: $x_{i}^{\prime}=x_{i}$.

In this model, we notice that the new state $x_{i}^{\prime}$ is given by the most represented state in the neighborhood. Individual expectations only play a role if there are more than one (most) representative state in the neighborhood. In this case, instead of choosing randomly any of these representative states, condition (5) is used to carry out the selection of the winning state. Either way, in contrast with the individual expectations dynamics, here the resulting updated value $x_{i}^{\prime}$ (if updated) will be the most represented state in the neighborhood.

\section{Lexicographical Energy Associated with the Dynamics}

In order to characterize and monitor the convergence of the dynamics of both models, we define the following quantities. First, let us consider the quantity that measures the level of mismatch of individual expectations with the attributes that a school offers, given by

$$
\mathscr{A}(\boldsymbol{x})=-\sum_{i=1}^{N} \boldsymbol{b}(i) \cdot \boldsymbol{a}\left(x_{i}\right) .
$$

Second, let us consider the Potts-like energy [34]:

$$
\mathscr{E}(\boldsymbol{x})=-\frac{1}{2} \sum_{i, j=1}^{N} w_{i j} \delta\left(x_{i}, x_{j}\right)
$$

where

$$
w_{i j}= \begin{cases}1 & \text { iff }(i, j) \in E \\ 0 & \text { otherwise }\end{cases}
$$


and

$$
\delta(a, b)=\left\{\begin{array}{ll}
1 & \text { iff } a=b \\
0 & \text { otherwise }
\end{array} .\right.
$$

We combine both quantities in the following vector:

$$
\mathscr{L}(\boldsymbol{x})=(\mathscr{A}(\boldsymbol{x}), \mathscr{E}(\boldsymbol{x})) .
$$

To analyze the vector quantity $\mathscr{L}(\boldsymbol{x})$, we consider the lexicographical order; i.e., given two real values, $u, v$, we say that a couple $(u, v)$ is lexicographically smaller than a couple $\left(u^{\prime}, v^{\prime}\right)$ if and only if $u<u^{\prime}$ or $u=u^{\prime}$ and $v<v^{\prime}$.

Suppose now that we updated the individual $i$ with the rule of the individual expectations dynamics (Section 2.1), so the new state configuration is $\boldsymbol{x}^{\prime}$ such that $x_{k}^{\prime}=x_{k} \forall k \neq i$ and $x_{i}^{\prime} \neq x_{i}$ such that $x_{i}^{\prime}$ is the new $i$-th state given by the local rule (1). Therefore, because the update is accomplished, one has $\boldsymbol{b}(i) \cdot \boldsymbol{a}\left(x_{i}^{\prime}\right)>\boldsymbol{b}(i) \cdot \boldsymbol{a}\left(x_{i}\right)$, then $\mathscr{A}\left(\boldsymbol{x}^{\prime}\right)<\mathscr{A}(\boldsymbol{x})$ and lexicographically $\mathscr{L}\left(\boldsymbol{x}^{\prime}\right)<\mathscr{L}(\boldsymbol{x})$. Notice that the condition $\mathscr{A}\left(\boldsymbol{x}^{\prime}\right)<\mathscr{A}(\boldsymbol{x})$ means that, as a consequence of the evolution by the rule, the vectors $\boldsymbol{b}(i)$ and $\boldsymbol{a}\left(x_{i}\right)$ become aligned (or mostly parallel) on average. This alignment represents essentially the mechanism of adaptation of the expectations.

Otherwise, if the update is conducted under the condition (3), i.e., $\boldsymbol{b}(i) \cdot \boldsymbol{a}\left(x_{i}^{\prime}\right)=\boldsymbol{b}(i) \cdot \boldsymbol{a}\left(x_{i}\right)$, then $\mathscr{A}\left(\boldsymbol{x}^{\prime}\right)=\mathscr{A}(\boldsymbol{x})$, but the new state $x_{i}^{\prime}$ is the most represented in the neighborhood. Since the $w_{i j}$ are symmetric, we have

$$
\begin{aligned}
\Delta \mathscr{E} & =\mathscr{E}\left(\boldsymbol{x}^{\prime}\right)-\mathscr{E}(\boldsymbol{x}) \\
& =-\sum_{j=1}^{N} w_{i j} \delta\left(x_{i}^{\prime}, x_{j}\right)+\sum_{j=1}^{N} w_{i j} \delta\left(x_{i}, x_{j}\right)<0 ;
\end{aligned}
$$

thus,

$$
\mathscr{E}\left(\boldsymbol{x}^{\prime}\right)<\mathscr{E}(\boldsymbol{x}) \Longrightarrow \mathscr{L}\left(\boldsymbol{x}^{\prime}\right)<\mathscr{L}(\boldsymbol{x}) .
$$

When an individual changes state, the vector $\mathscr{L}$ lexicographically decreases strictly. So $\mathscr{L}$ can be considered as a kind of Lyapunov function (decreasing monotone function) driven by the network dynamics. Moreover, we may bound the number of steps needed to reach a fixed point. In fact, we have

$$
\begin{aligned}
-s N & \leq \mathscr{A}(\boldsymbol{x}) \leq 0 \\
-\frac{\|W\|}{2} N^{2} & \leq \mathscr{E}(\boldsymbol{x}) \leq 0,
\end{aligned}
$$

where $s$ is the number of attributes and $\|W\|=$ $(1 / N) \sum_{i, j}\left|w_{i j}\right| \leq 1$. Therefore,

$$
-\left(s N, \frac{\|W\|}{2} N^{2}\right) \leq \mathscr{L}(\boldsymbol{x}) \leq(0,0) ;
$$

thus, $\mathscr{L}(\boldsymbol{x})$ is lexicographically strictly decreasing and bounded. For any initial state configuration the asynchronous dynamics converges to a fixed point (every site remains invariant) at most in $\sim \mathcal{O}\left(N^{2}\right)$ steps.
For the social expectations dynamics (Section 2.2), where the update value is given essentially by the most represented state in the neighborhood, we have shown that for this case $\mathscr{E}\left(\boldsymbol{x}^{\prime}\right)<\mathscr{E}(\boldsymbol{x})$. Therefore, by interchanging the order of the components of the vector

$$
\widetilde{\mathscr{L}}(\boldsymbol{x})=(\mathscr{E}(\boldsymbol{x}), \mathscr{A}(\boldsymbol{x})),
$$

we have the same convergence as above, that is, $\widetilde{\mathscr{L}}\left(\boldsymbol{x}^{\prime}\right)<$ $\widetilde{\mathscr{L}}(\boldsymbol{x})$.

So, we conclude that both strategies, the individual expectations model and the social expectations model, converge to fixed points, yet not necessarily at the same speed nor to the same fixed point.

In the next section, we develop simulations for those dynamics. For illustrative purposes, the states in the previous models will be seen as schools having some attributes.

\section{Simulations of the Two Strategies}

In this section, we compare the convergence to fixed points of the two strategies (individual and social expectations) for different configurations of the schools' attribute vector $\boldsymbol{a}$ and the individual expectations vector $\boldsymbol{b}$. The simulations presented below were done using a squared two-dimensional regular lattice of $64 \times 64$ nodes with periodic boundary conditions. Each individual is connected with the closest neighbor in a Moore vicinity. In both cases, i.e., individual and social expectations, we consider the number of states $q=100$ and a number of attributes ranging in $s \in\{2,10\}$. We randomly explore $\boldsymbol{a}$ and $\boldsymbol{b}$ as follows. With probability $p_{a}$, a school attribute is set to 1 . That is, a school has on average $s p_{a}$ positive attributes. Similarly, an individual expects a specific attribute with probability $p_{b}$. Hence, she demands on average $s p_{b}$ attributes. We vary $p_{a}$ from 0.01 to 0.99 with a step increase of 0.0125 , and we consider $p_{b}=1-p_{a}$. Once the dynamics converges to a solution (fixed point), we analyze the fraction of schools that have been selected, or equivalently, the fraction of schools with students.

4.1. The Dynamics of Individual Expectations. Numerical simulations of the individual expectation models (Section 2.1) show different scenarios. For $s=2$, Figure 1 shows a transition from only a few schools with students to almost all the schools with students as $p_{a} \longrightarrow 1$ (thus, $p_{b} \longrightarrow 0$ ). With a critical transition threshold at $p_{a}^{*}=p_{c} \approx 0.45$, it is more likely that, for values of $p_{a}>p_{c}$, all (or most) of the schools will be selected. On the other hand, for $p_{a}<p_{c}$ only a few schools will be selected. In this last case, we have that all of the $64 \times 64=4096$ individuals (families) select a few schools. Therefore, if we consider that in reality schools have a fixed capacity, in practice there would be students left out. Figure 2 considers and example when $p_{a}<p_{c}$. In particular, Figure 2(a) shows the spatial patterns for the fixed point solution when $s=2$ and $p_{a}=0.25$ and Figure 2(b) the distribution of the number of students per school. As expected, only few schools satisfy the individual expectations as $p_{b}$ increases $\left(p_{b}=0.75\right)$. Since individuals request a large number of attributes, only a small number of schools 


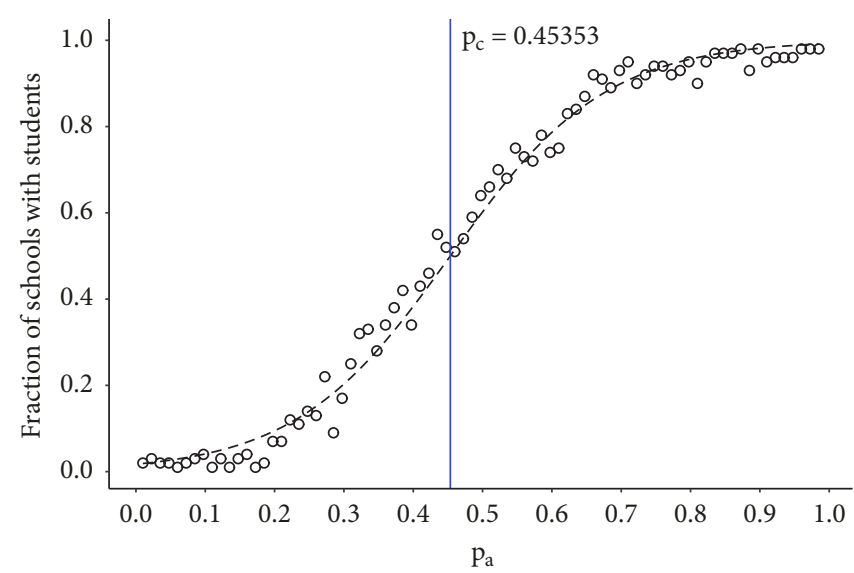

Figure 1: The fraction of schools with students for different values of $p_{a}$, under the individual expectations model. The segmented line corresponds to the fit with $\left(1+e^{-\beta\left(p_{a}-p_{c}\right)}\right)^{-1}$ with $\beta=8.9$ and $p_{c}=0.45$.

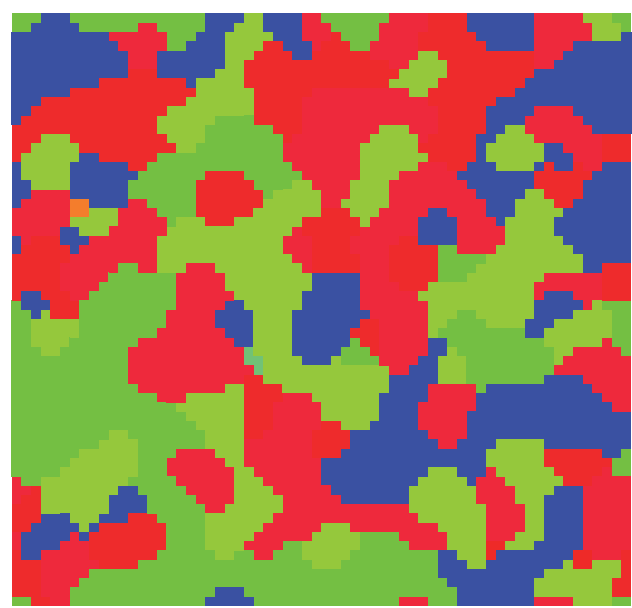

(a)

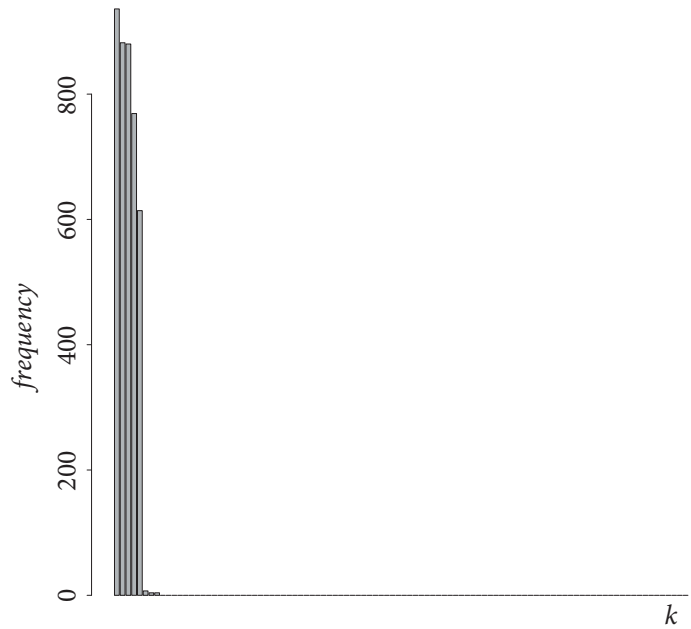

(b)

Figure 2: Example of a fixed point solution for $s=2, p_{a}=0.25$, and $p_{b}=0.75$. (a) The spatial patterns of selected schools (the colormap labels the states $\{1,2, \ldots 100\})$. (b) The distribution of the number of students per school.

can match individual expectations. The distribution of the number of students per provider is strongly localized into a reduced number of schools.

On the other hand, Figure 3 considers an example when $p_{a}>p_{c}$, in particular, for $s=2$ and $p_{a}=0.75$. We observe that the concentration of preferences on a reduced number of schools changes as the intensity of individual expectations decreases, namely, $p_{b}=0.25$. In other words, when the number of attributes is small and individual expectations are relaxed, more schools satisfy the individual expectations.

When we consider $s=10$, we obtain a transition and a critical threshold $\left(p_{a}^{*}=p_{c} \approx 0.47\right)$ similar to Figure 1. Figure 4 is an example with the parameter settings of Figure 2 but considers $s=10$. We observe the most extreme case of concentration of preferences. Considering a real-case scenario, this would mean that a number of families is left unattended by the prevailing school offer, and that most of the schools receive no preferences from parents.

Finally, Figure 5 is an example with the parameter settings of Figure 3, now with $s=10$. We observe that the strong concentration of preferences on a reduced number of schools disappears as individual expectations become more relaxed.

The main observation of this simulation is that the intensity in which individuals expect their personal beliefs, desires, and needs (individual expectations) to be satisfied by providers is a crucial factor for a better matching between school offer and parental preferences. The diversity of schools attributes (either $s=2$ or 10 ) is a secondary factor affecting this matching.

4.2. The Dynamics of Social Expectations. Here we repeat the same simulations as before, but using the social expectations 


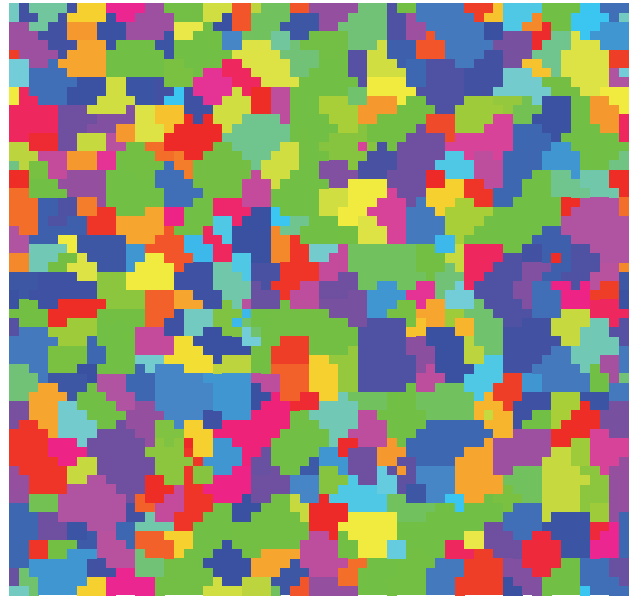

(a)

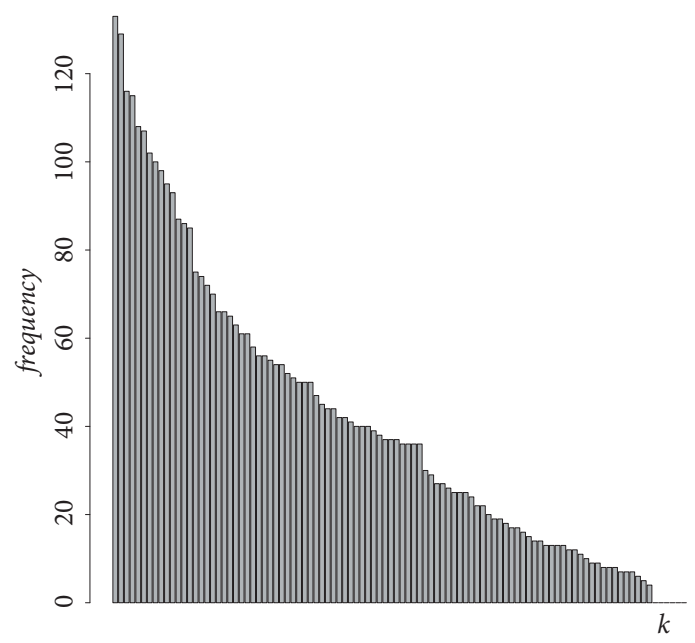

(b)

Figure 3: Example of a fixed point solution for $s=2, p_{a}=0.75$, and $p_{b}=0.25$. (a) The spatial patterns of selected schools (the colormap labels the states $\{1,2, \ldots 100\}$ ). (b) The distribution of the number of students per school.

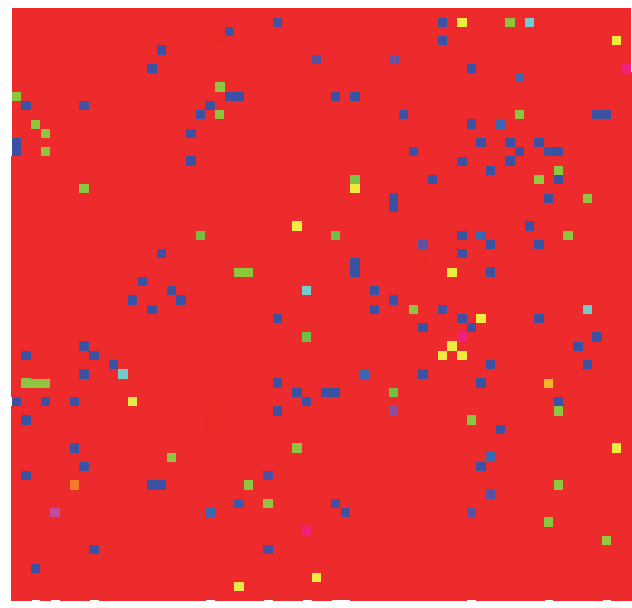

(a)

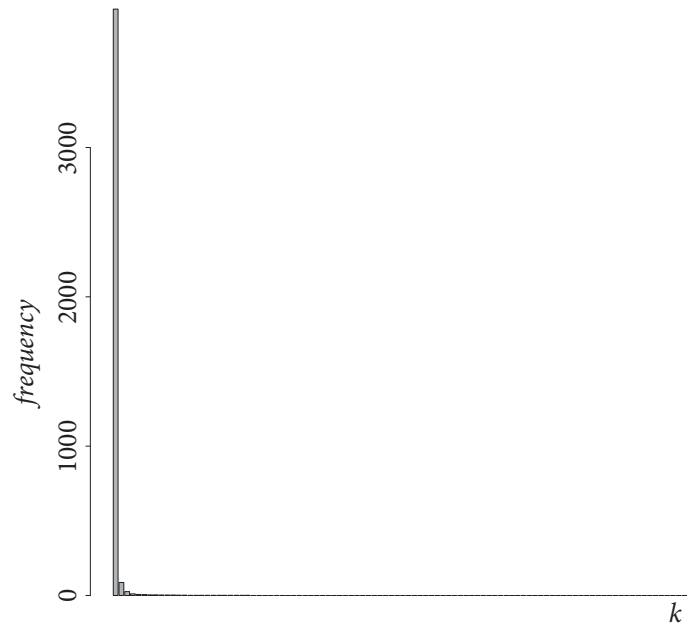

(b)

FIGURE 4: Example of a fixed point solution for $s=10, p_{a}=0.25$, and $p_{b}=0.75$. (a) The spatial patterns of selected schools (the colormap labels the states $\{1,2, \ldots 100\}$ ). (b) The distribution of the number of students per school.

model. For $s=2$, Figure 6 shows that there is practically no transition as $p_{a} \longrightarrow 1$ (thus, $p_{b} \longrightarrow 0$ ). This is interesting, since we can appreciate the effect of previous social interactions and the scanning of aggregated preferences in the network vicinity, especially for individuals with high expectations, where now, they will most likely end up matching with a school.

For $s=2$, Figure 7 shows an example for $p_{a}=0.25$, and Figure 8 shows an example for $p_{a}=0.75$. When we consider $s=10$, we observe a similar behavior as for $s=2$, i.e., no transition. In this case $(s=10)$, Figure 9 shows an example for $p_{a}=0.25$, and Figure 10 shows an example for $p_{a}=0.75$. As seen, for $p_{b}=0.75$ (Figures 7(b) and 9(b)), we found cases with some concentration of preferences but not as extreme as in the individual expectations dynamics with similar settings. For $p_{b}=0.25$ (Figures $8(\mathrm{~b})$ and 10(b), more relaxed expectations) the number of schools satisfying social preferences increases. Hence, with the social expectations model, we obtain a more distributed market environment in which different schools satisfy social expectations.

As expected, we see from the examples that $p_{a}$ or $p_{b}$ do not have a significant impact on the fraction of schools with students, but more on the distribution of students per school.

\section{Discussion}

Connecting the results of our simulations with some relevant topics in the empirical literature of schools choices, we can establish some interesting insights. 


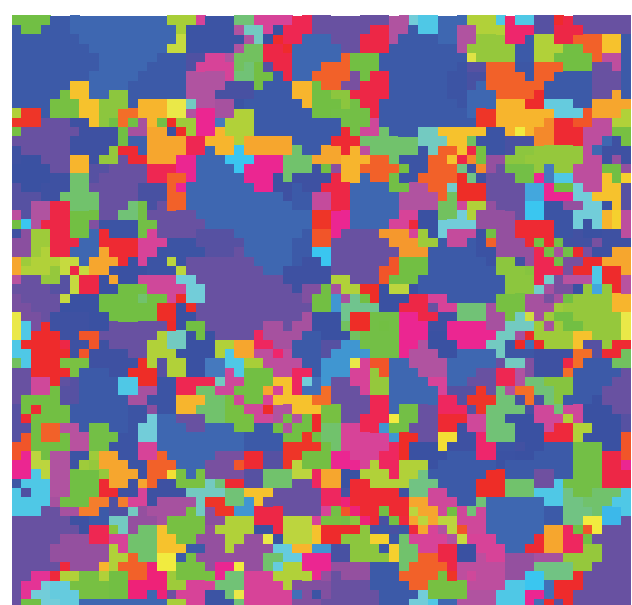

(a)

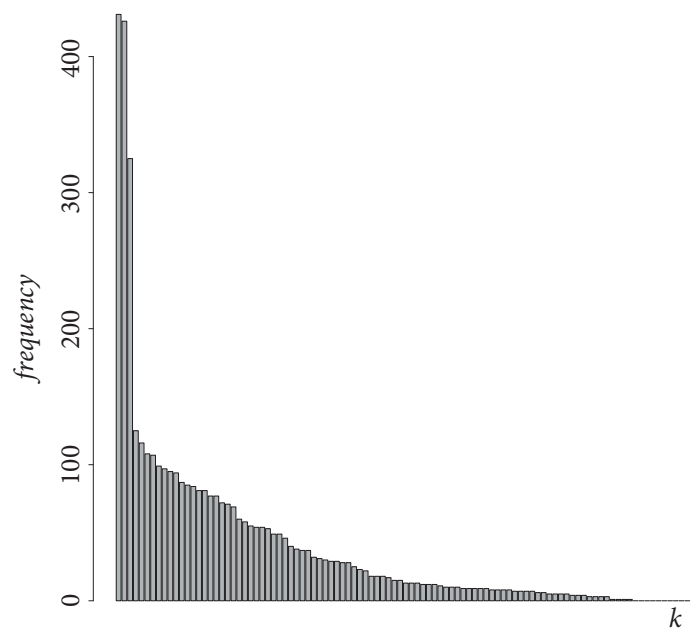

(b)

Figure 5: Example of a fixed point solution for $s=10, p_{a}=0.75$, and $p_{b}=0.25$. (a) The spatial patterns of selected schools (the colormap labels the states $\{1,2, \ldots 100\}$ ). (b) The distribution of the number of students per school.

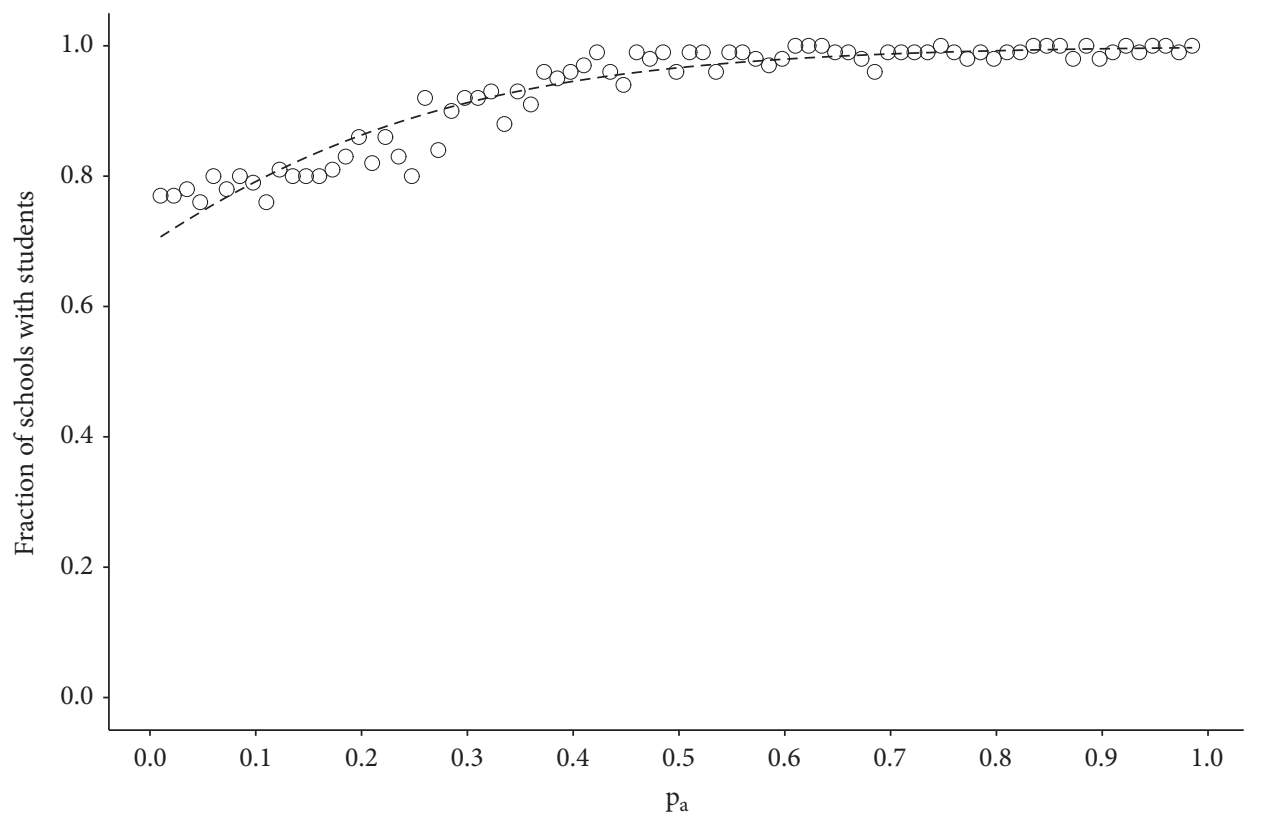

Figure 6: The fraction of schools with students for different values of $p_{a}$, under the social expectations model.

Our main contention is that by relying only on individual expectations (parents' strong beliefs, desires, and needs), the system experiences an unstable dynamics characterized by a nonlinear transition of probabilistic origins. In this complex behavior "a positive feedback pushes a runaway change to a contrasting state once a threshold is passed" ([32]: 104). In our case, the persistence on individual preferences in school choice decisions (positive feedback loop, Figure 1) works as a lock-in mechanism of systems' behavior [35] that leads with $p_{a}<0.45$ (critical threshold for $s=2$ ) to an over-concentration of demand in few schools that meet those heterogeneous preferences. A lock-in mechanism is caused by a "self-reinforcing adherence to a mode of behavior [that] tends to promote inertia, a lack of responsiveness to changes in the environment" ([32]: 245). In our case, this self-reinforcing adherence is represented by the persistence of parents selecting schools that perfectly match with their own beliefs, desires, and needs, even once the capacity of schools for attending children is surpassed. The over-concentration of demand and consequently the fact that in this case a number of families would be left unattended by the prevailing school offer push the system to another probabilistic state $\left(p_{a}>0.45\right.$ for $s=2$ ). In this state, each school receives preferences from parents. The system becomes thus unstable: beyond the critical threshold, it undergoes reorganization and moves to another steady state. 


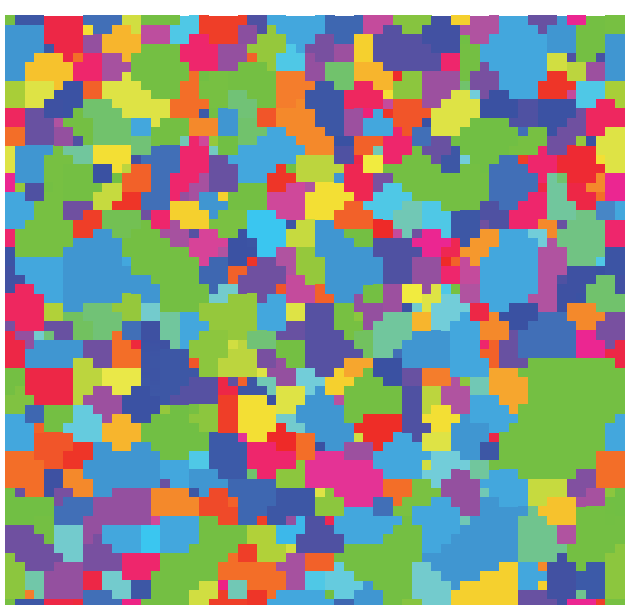

(a)

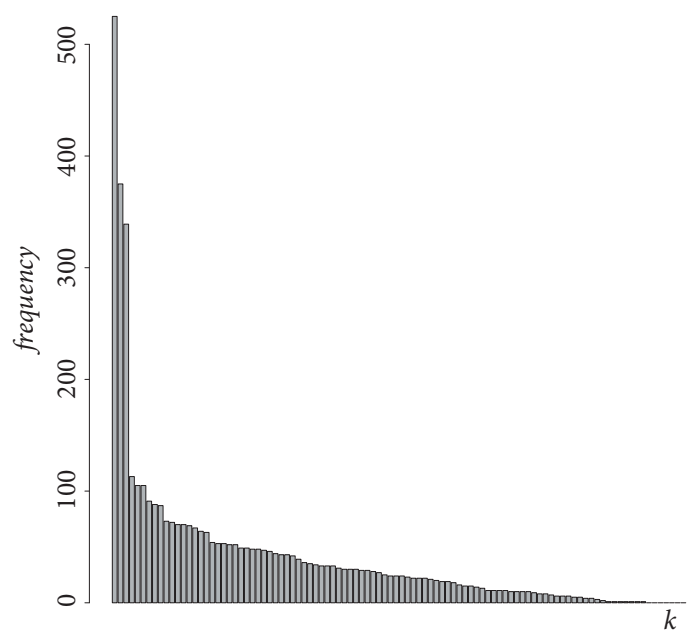

(b)

Figure 7: Example of a fixed point solution for $s=2, p_{a}=0.25$, and $p_{b}=0.75$. (a) The spatial patterns of selected schools (the colormap labels the states $\{1,2, \ldots 100\}$ ). (b) The distribution of the number of students per school.

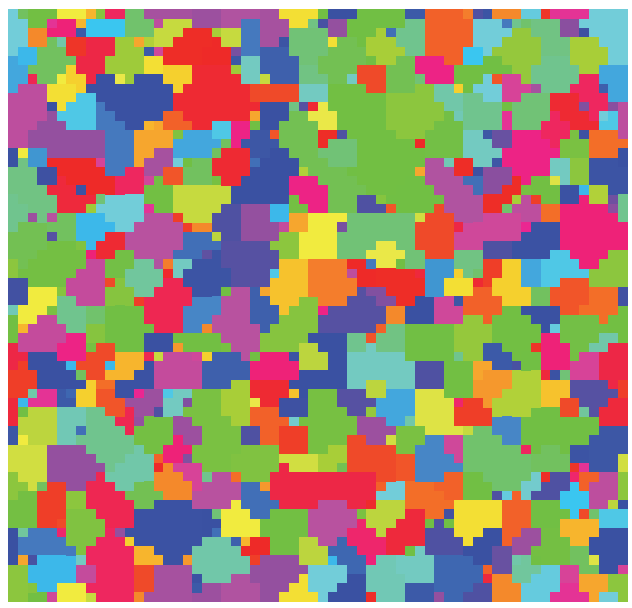

(a)

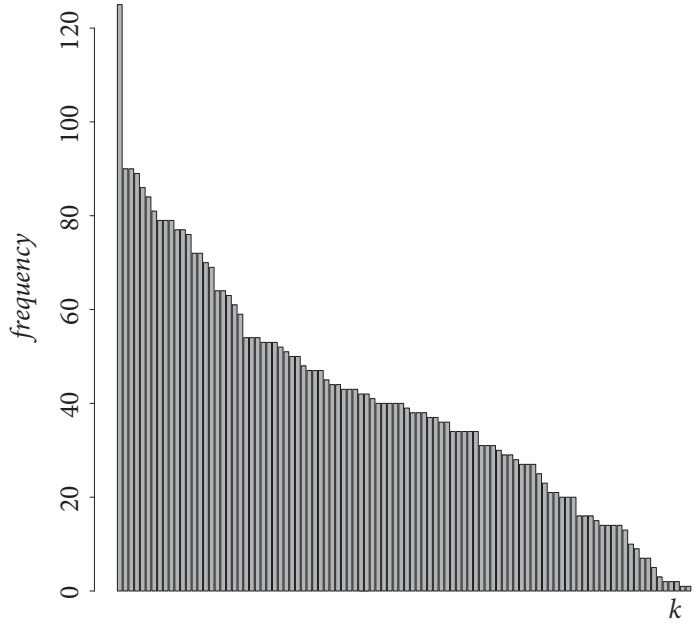

(b)

FIGURE 8: Example of a fixed point solution for $s=2, p_{a}=0.75$, and $p_{b}=0.25$. (a) The spatial patterns of selected schools (the colormap labels the states $\{1,2, \ldots 100\}$ ). (b) The distribution of the number of students per school.

On the contrary, when the school choice relies on social expectations (the most represented state in the neighborhood of the selected individual), the system shows a rather stable dynamics in which all the schools receive preferences by parents. One fundamental presupposition of school choice is that parents rely on a rational model of behavior that considers only individual expectations organized in terms of costs, benefits, information, and opportunities [10, 20]. Our simulations show that this model of decision-making (individual expectations) converges to alternative fixed points at the cost of systemic instability characterized by a probabilistic transition once the critical threshold has been reached (Figure 1). Instead, no transition appears in the social expectations model. This model positions parents in a rather sociohistorical context (networks, relations, shared experiences, social memory). It represents a socially more efficient situation, since by primarily scanning the preferences in the social landscape and secondarily the personal experience (in case of a tie, o more concretely put, in case of indecision), individuals may choose schools by giving priority to the information provided by their networks considering the position one has in a particular social setting (what can I realistically aspire to?) and the eligible schools according to it (what is the hierarchy of priorities?).

As we know from the literature, one major motivation of parents regarding school choice is to improve the outcomes of their children by accessing to higher quality schools and to a better match between school offer and parental preferences [18]. Our simulations show that increasing the social interaction with the neighborhood is the best option for an 


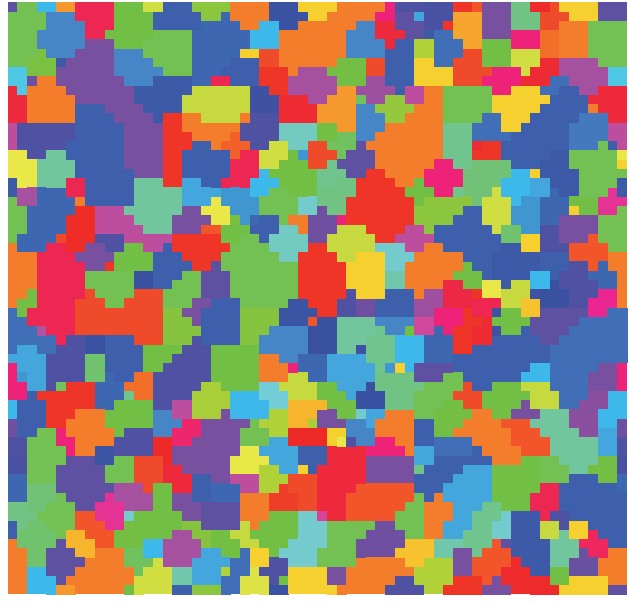

(a)

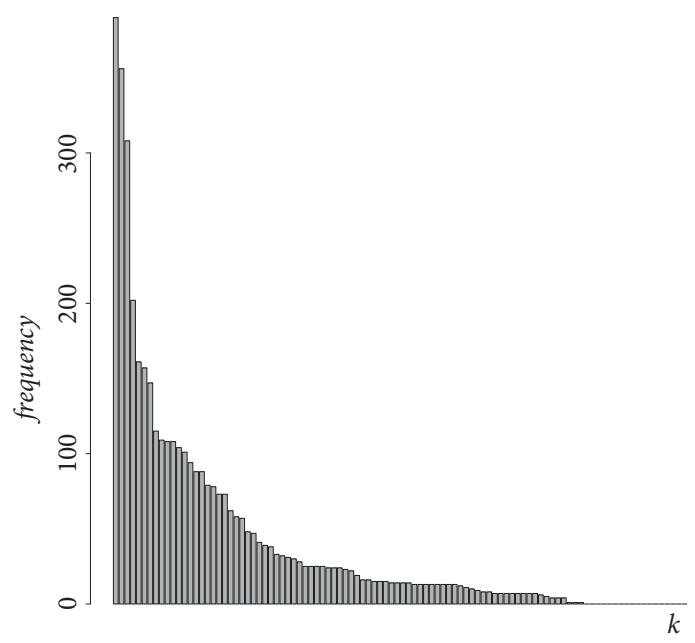

(b)

Figure 9: Example of a fixed point solution for $s=10, p_{a}=0.25$, and $p_{b}=0.75$. (a) The spatial patterns of selected schools (the colormap labels the states $\{1,2, \ldots 100\})$. (b) The distribution of the number of students per school.

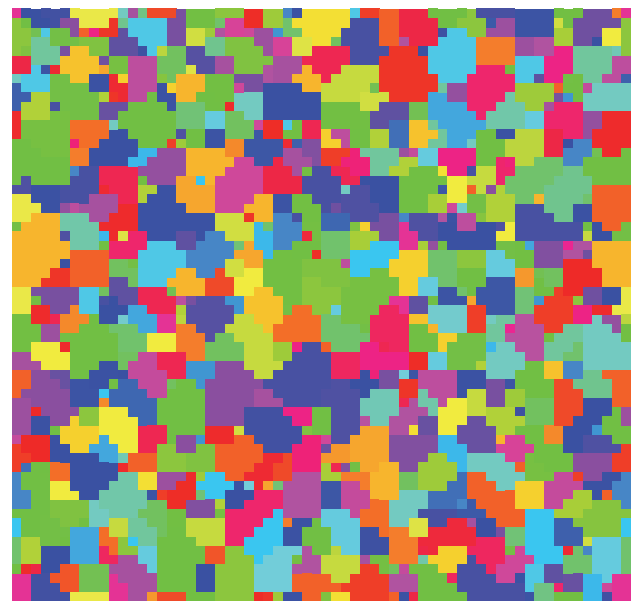

(a)

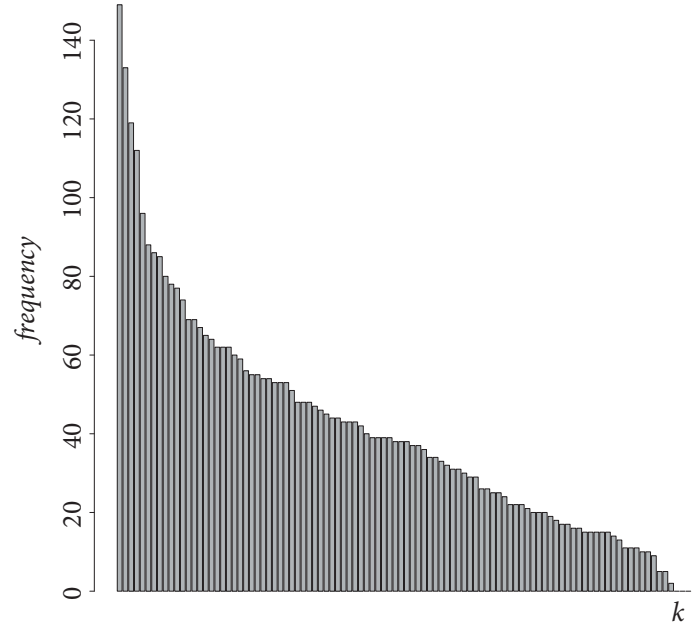

(b)

Figure 10: Example of a fixed point solution for $s=10, p_{a}=0.75$, and $p_{b}=0.25$. (a) The spatial patterns of selected schools (the colormap labels the states $\{1,2, \ldots 100\}$ ). (b) The distribution of the number of students per school.

improved matching. If individual expectations are too strong (i.e., parents do not need persistent interaction because their beliefs, desires, and needs are the only thing that matters), the system runs the risk of over-concentration of demand in a few schools located below the critical threshold (Figure 1, $p_{a}<$ $\left.p_{c}\right)$. On the contrary, when individual expectations are more relaxed-i.e., over the critical threshold in Figure $1\left(p_{a}>\right.$ $p_{c}$ ), or when we consider social expectations (Figure 6)-a distributed pattern of school choice and parental preferences appears. In other words, by scanning the social landscape (network vicinity) individuals may adjust their preferences so that different schools can meet their adaptive requirements. The social landscape becomes thus a way of individuals to reflect upon and change their own beliefs, desires, and needs.
In addition to parents preferences, availability of schools, defined as the existence of schools in a given district, and accessibility, defined as the probability of families to be accepted by schools, are also relevant conditions of school choice [12]. Considering our simulations, Figure 1 (individual expectations) shows lower conditions for accessibility particularly below the critical threshold $\left(p_{a}<p_{c}\right)$, irrespective of whether schools exist in the neighborhood or not. On the contrary, accessibility significantly increases in Figure 6 (social expectations) for all individuals (families). In this sense, for improving accessibility -and, therefore, for parents to perform a better matching between school offer and preferences in a market environment- social expectations seems to be a primary factor to be taken into consideration. 
Competition for the top schools is an operational condition in a market environment that allegedly improves quality of schools services and leads to better outcomes [6]. When we consider Figure 1, and particularly Figures 2(a) and 2(b) (individual expectations below the critical threshold, $p_{a}<p_{c}$ ), competition is reduced to an insufficient number of schools, while others are excluded from competition and, therefore, from its eventual positive effects. In this case, competition among schools depends on individuals relaxing their preferences (particularly Figures 3(a) and 3(b)). Alternatively, when school choice takes into account social expectations (Figure 6), incentives for schools to compete for (better) students increase, since every school may receive a significant fraction of students. Additionally, "schools cannot allow themselves to be perceived as ordinary[,] so they must represent themselves as being especially successful, distinctive, and desirable" [11] (page 405). Since our model of social expectations considers individual expectations in case of a tie of states in the neighborhood, more and better students and a better matching of parental preferences are relevant factors that reinforce the need of schools for a positive self-representation regarding competition.

Broadly speaking, our simulations support views on school choice in market environments that consider the relevance of scanning and interacting with the relevant social landscape (network vicinity) for a better informed decisionmaking process $[8-10,33,36]$. In so doing, individuals can combine strategies of competition among unknown individuals with forms of cooperation with the proximate network. Furthermore, they can obtain better information about particular schools (old and new) and gather multiple perspectives on a single school. Subsequently, with this improved information from their networks, they can also design strategies aimed at increasing their social capital by selecting schools preferred by groups of higher socioeconomic standing. As a consequence, by relying primarily on social expectations and secondarily (in case of a tie of most representative states in the neighborhood) on individual expectations, the rather individualistically oriented market of school choice may turn into a more public oriented landscape.

\section{Conclusion}

We have analyzed two simple dynamical models of decision that represent the school choice problem under two views, one that focuses on individual expectations when deciding for a school, without major consideration of the social environment in which the decision is made, and the other focused on social expectations modeled by the neighborhood preferences when deciding for a school. Through computational simulations, we found a probabilistic transition (and a critical threshold) on the number of schools with students in the individual expectations model: when $p_{a}<0.45$ the demand concentrates in a few schools; when $p_{a}>0.45$ most of the schools receive students, whereas for the social expectations model, we found no transition. Thus, most of the schools will have students all the time. In this case, the probability parameters played a more relevant role in the number of students per school.

In relation to key topics highlighted by the school choice literature, our simulations show that the social expectations model (scanning the social landscape for network relations, shared experiences, social memory, and information) represents a more socially efficient situation that may help individuals (parents, families) to increase their options of accessibility, information, social capital, and improved school performances. As a consequence, a better matching between school offer and parental preferences arises that contribute to a more public orientation of school choice in a market environment.

We focused in this paper on the problem of school choice as an illustration of the performance of the models in a complex social environment. However, the models and the emergent dynamics they bring to light are applicable to other fields in social sciences in which nonlinear behavior might appear. Political elections particularly in two-round systems comparing first election and ballotage, international relations especially in cases of complex coordinations and negotiations with many layers and actors, or economic decisions regarding the exploration of new productive landscapes and the innovation with novel technologies, are possible social fields in which the operation of the two-level decision rule (individual decision versus majority voting, or individual versus social expectations) may show its applicability as in the analyzed case of school choice in a market environment.

\section{Data Availability}

No data were used to support this study.

\section{Conflicts of Interest}

The authors declare that they have no conflicts of interest.

\section{Acknowledgments}

This work was supported by the Research Center Millennium Nucleus Models of Crises NS130017 (Chile) and the Dirección de Investigación at Universidad Adolfo Ibáñez, CONICYTPFCHA/Doctorado Nacional/2017-21170290 (Catalina Canals), Basal Project CMM (Gonzalo A. Ruz, Eric Goles, and Sergio Rica), ECOS C16E01 (Eric Goles and Gonzalo A. Ruz), and the supercomputing infrastructure of the NLHPC (ECM-02) at the FCFM, Universidad de Chile. We would like to thank CONICYT-Chile under grant Fondecyt 1181382 (Sergio Rica) and Fondecyt 1180706 (Gonzalo A. Ruz).

\section{References}

[1] A. Chong and F. López, Privatization in Latin America: Myths and Reality, Stanford University Press, Stanford, Calif, USA, 2005.

[2] R. Kemp, Privatization: The Provision of Public Services by the Private Sector, McFarland \& Co, Jefferson, NC, USA, 2007. 
[3] J. Gould, Politics of Privatization: Wealth and Power in Postcommunist Europe, Lynne Rienner Publishers, Boulder, Colo, USA, 2011.

[4] A. Verger, C. Fontdevila, and A. Zancajo, The Privatization of Education: A Political Economy of Global Education Reform, Teachers College Press, New York, NY, USA, 2016.

[5] J. E. Chubb and T. M. Moe, "America’s Public Schools: Choice Is a Panacea," The Brookings Review, vol. 8, no. 3, pp. 4-12, 1990.

[6] C. Teelken, "Market mechanisms in education: School choice in The Netherlands, England and Scotland in a comparative perspective," Comparative Education, vol. 35, no. 3, pp. 283-302, 1999.

[7] P. Woods, C. Bagley, and R. Glatter, School Choice and Competition: Markets in The Public Interest? Routledge, New York, NY, USA, 1998.

[8] C. Bagley, "School choice and competition: A public-market in education revisited," Oxford Review of Education, vol. 32, no. 3, pp. 347-362, 2006.

[9] D. J. Ferrero, "Fresh perspectives on school choice," Journal of Philosophy of Education, vol. 38, no. 2, pp. 287-296, 2004.

[10] C. A. Bell, "All choices created equal? The role of choice sets in the selection of schools," Peabody Journal of Education, vol. 84, no. 2, pp. 191-208, 2009.

[11] L. Angus, "School choice: neoliberal education policy and imagined futures," British Journal of Sociology of Education, vol. 36, no. 3, pp. 395-413, 2015.

[12] J. Lee, "Paying for school choice: availability differences among local education markets," International Journal of Education Policy and Leadership, vol. 11, no. 5, 2016.

[13] H. Santos and G. Elacqua, "Socioeconomic school segregation in Chile: Parental choice and a theoretical counterfactual analysis," CEPAL Review, vol. 2016, no. 119, pp. 123-137, 2017.

[14] T. Moe and J. Chubb, Liberating Learning. Technology, Politics, and the Future of American Education, John Wiley \& Sons, San Francisco, Calif, USA, 2006.

[15] A. J. Egalite, "Measuring competitive effects from school voucher programs: a systematic review," Journal of School Choice, vol. 7, no. 4, pp. 443-464, 2013.

[16] R. Chakrabarti, "Vouchers, public school response, and the role of incentives: Evidence from florida," Economic Inquiry, vol. 51, no. 1, pp. 500-526, 2013.

[17] C. A. DeAngelis, "Do self-interested schooling selections improve society? A review of the evidence," Journal of School Choice, vol. 11, no. 4, pp. 546-558, 2017.

[18] C. A. DeAngelis and H. H. Erickson, "What leads to successful school choice programs? A review of the theories and evidence," the Cato Journal, vol. 38, no. 1, pp. 247-263, 2018.

[19] N. Luhmann and K. E. Schorr, Reflexion im Erziehungssystem, Suhrkamp, Frankfurt, Germany, 1999.

[20] S. R. Ben-Porath, "School choice as a bounded ideal," Journal of Philosophy of Education, vol. 43, no. 4, pp. 527-544, 2009.

[21] J. Coleman, Foundations of Social Theory, Harvard University Press, Cambridge, Mass, USA, 1990.

[22] C. Fershtman and U. Segal, "Preferences and Social Influence," American Economic Journal: Microeconomics, vol. 10, no. 3, pp. 124-142, 2018.

[23] N. Luhmann, Theory of Society, Stanford University Press, Stanford, Calif, USA, 2012.

[24] C. Moore, "Majority-vote cellular automata, Ising dynamics, and P-completeness," Journal of Statistical Physics, vol. 88, no. 3-4, pp. 795-805, 1997.
[25] E. Goles, P. Montealegre, K. Perrot, and G. Theyssier, "On the complexity of two-dimensional signed majority cellular automata," Journal of Computer and System Sciences, vol. 91, pp. $1-32,2018$.

[26] E. Goles and S. Martínez, Neural and Automata Networks: Dynamical Behavior and Applications, vol. 58 of Mathematics and Its Applications, Kluwer Academic, Dordrecht, Netherlands, 1990.

[27] A. Krawiecki, "Spin-glass-like transition in the majority-vote model with anticonformists," The European Physical Journal B, vol. 91, no. 3, 2018.

[28] D. Kempe, A. Dobra, and J. Gehrke, "Gossip-based computation of aggregate information," in Proceedings of the 44th Annual IEEE Symposium on Foundations of Computer Science (FOCS '03), pp. 482-491, Cambridge, Mass, USA, October 2003.

[29] E. Mossel, J. Neeman, and O. Tamuz, "Majority dynamics and aggregation of information in social networks," Autonomous Agents and Multi-Agent Systems, vol. 28, no. 3, pp. 408-429, 2014.

[30] T. Schelling, Micromotives and Macrobehavior, W. W. Norton \& Company, New York, NY, USA, 2006.

[31] P. Medina, E. Goles, R. Zarama, and S. Rica, "Self-organized societies: on the sakoda model of social interactions," Complexity, vol. 2017, Article ID 3548591, 16 pages, 2017.

[32] M. Scheffer, Critical Transitions in Nature and Society, Princeton University Press, Princeton, NJ, USA, 2009.

[33] C. Koedel, J. R. Betts, L. A. Rice, and A. C. Zau, "The integrating and segregating effects of school choice," Peabody Journal of Education, vol. 84, no. 2, pp. 110-129, 2009.

[34] R. B. Potts, "Some generalized order-disorder transformations," Mathematical Proceedings of the Cambridge Philosophical Society, vol. 48, no. 1, pp. 106-109, 1952.

[35] M. Scheffer and F. R. Westley, "The evolutionary basis of rigidity: Locks in cells, minds, and society," Ecology and Society, vol. 12, no. 2, 2007.

[36] L. Angus, "School Choice: Shaping Personal, Family and Social Imaginaries in an Era of Neoliberalism," Literacy Information and Computer Education Journal, vol. 3, no. 1, pp. 621-629, 2012. 


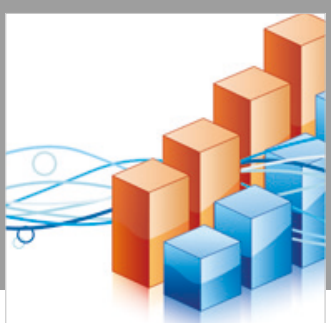

Advances in

Operations Research

\section{-n-m}
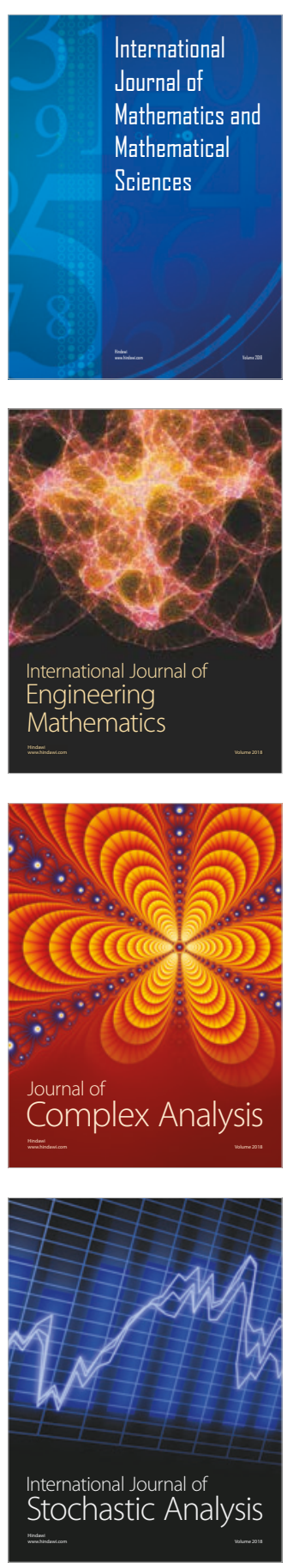
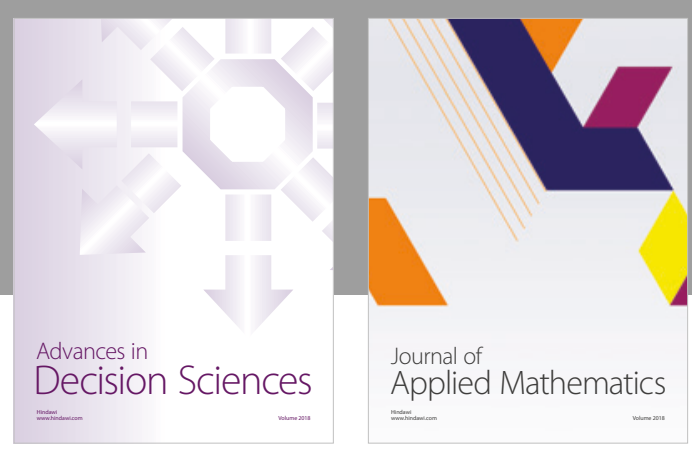

Journal of

Applied Mathematics
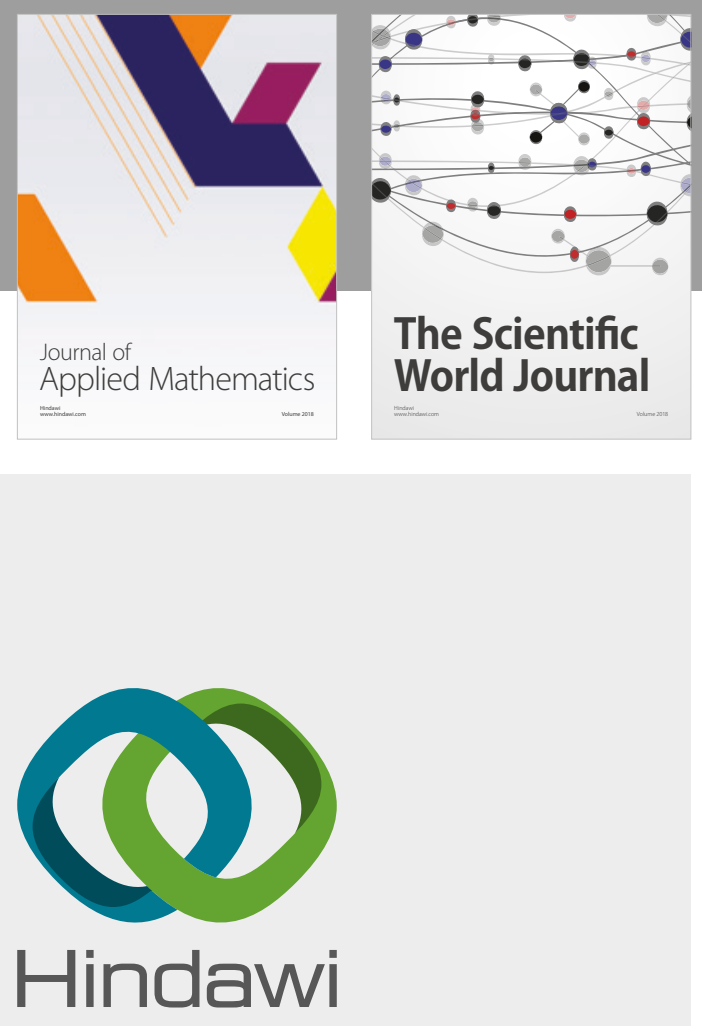

Submit your manuscripts at

www.hindawi.com

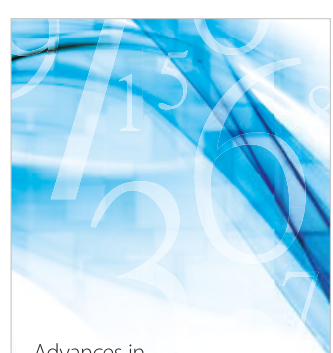

Advances in
Numerical Analysis
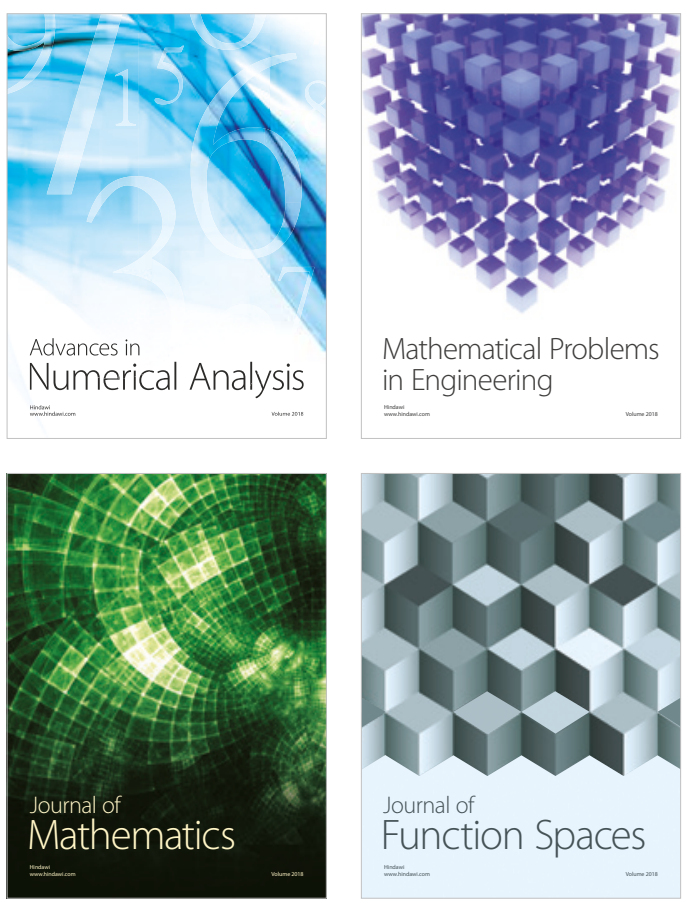

Mathematical Problems in Engineering

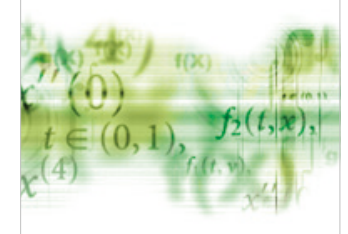

International Journal of

Differential Equations

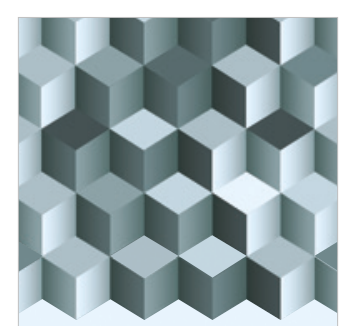

Journal of

Function Spaces
The Scientific

World Journal

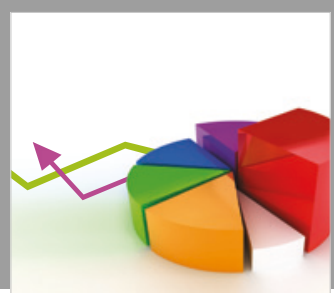

Journal of

Probability and Statistics
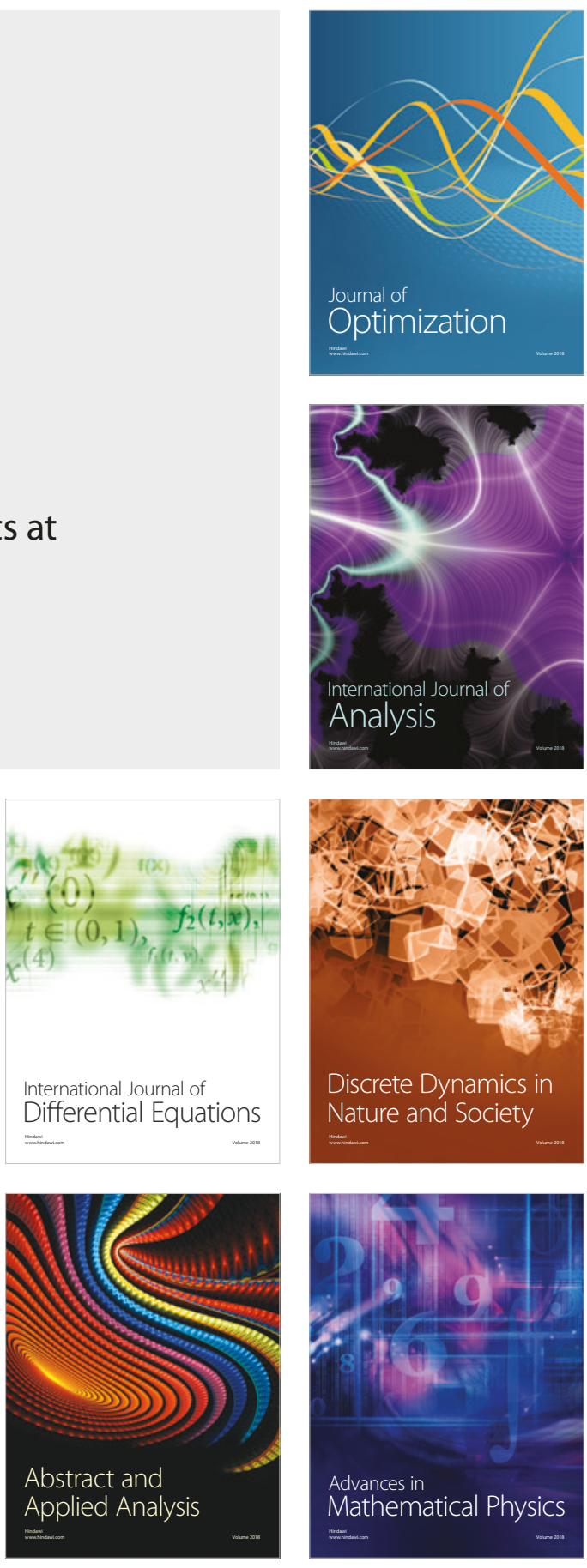